\title{
RESEARCH
}

Open Access

\section{Reproducibility of foot dimensions measured from 3-dimensional foot scans in children and adolescents with Down syndrome}

\author{
Nirmeen M. Hassan ${ }^{1,2^{*}}$ (D), Andrew K. Buldt ${ }^{1,3}$, Nora Shields ${ }^{2,3,4}$, Karl B. Landorf ${ }^{1,2,3}$, Hylton B. Menz ${ }^{1,3}$ and
} Shannon E. Munteanu ${ }^{1,3}$

\begin{abstract}
Background: Children and adolescents with Down syndrome have a distinctive foot shape (such as wide and flat feet) that often leads to difficulty with footwear fitting. 3-dimensional (3D) scanning can accurately measure the foot dimensions of individuals with Down syndrome, which may assist shoe fit. However, the reproducibility of measuring foot dimensions using 3D scans in children and adolescents with Down syndrome is unknown. The aim of this study was to determine the intra- and inter-rater reproducibility of measuring foot dimensions of children and adolescents with Down syndrome using 3D scanning.

Methods: 3D foot scans of 30 participants with Down syndrome aged 5 to 17 years were obtained using the FotoScan 3D scanner. Foot dimensions assessed were foot length, ball of foot length, outside ball of foot length, diagonal foot width, horizontal foot width, heel width, ball girth, instep girth, first and fifth toe height, and instep height. Additionally, the Wesjflog Index and forefoot shape were determined. Measurements were completed by two raters independently on two separate occasions, 2 weeks apart. Intra- and inter-rater reliability were assessed using intra-class coefficients (ICCs) and Gwet's AC1 statistics with 95\% confidence intervals. Agreement was determined by calculating limits of agreement (LOA) and percentage agreement.
\end{abstract}

Results: Eighteen participants were female and 12 were male (mean age 10.6 [3.9] years). Intra-rater reproducibility (ICCS ranged from 0.74 to $0.99,95 \%$ LOA from $-13.7 \mathrm{~mm}$ to $16.3 \mathrm{~mm}$ ) and inter-rater reproducibility (ICCs ranging from 0.73 to $0.99,95 \%$ LOA from - $18.8 \mathrm{~mm}$ to $12.7 \mathrm{~mm}$ ) was good to excellent, although some measurements (ball of foot length, outside ball of foot length, heel width and girth measurements) displayed wider LOAs indicating relatively poorer agreement. Forefoot shape displayed substantial to almost perfect reliability (Gwet's AC1 0.68 to 0.85 ) and percentage agreement ranged from 73 to $87 \%$, indicating acceptable agreement.

(Continued on next page)

\footnotetext{
* Correspondence: n.hassan@latrobe.edu.au

'Discipline of Podiatry, School of Allied Health, Human Services and Sport, La Trobe University, Victoria 3086, Australia

2Living with Disability Research Centre, School of Allied Health, Human Services and Sport, La Trobe University, Victoria 3086, Australia

Full list of author information is available at the end of the article
}

(c) The Author(s). 2020 Open Access This article is licensed under a Creative Commons Attribution 4.0 International License, which permits use, sharing, adaptation, distribution and reproduction in any medium or format, as long as you give appropriate credit to the original author(s) and the source, provide a link to the Creative Commons licence, and indicate if changes were made. The images or other third party material in this article are included in the article's Creative Commons licence, unless indicated otherwise in a credit line to the material. If material is not included in the article's Creative Commons licence and your intended use is not permitted by statutory regulation or exceeds the permitted use, you will need to obtain permission directly from the copyright holder. To view a copy of this licence, visit http://creativecommons.org/licenses/by/4.0/ The Creative Commons Public Domain Dedication waiver (http://creativecommons.org/publicdomain/zero/1.0/) applies to the data made available in this article, unless otherwise stated in a credit line to the data. 
(Continued from previous page)

Conclusions: The measurement of specific foot dimensions of children and adolescents with Down syndrome using 3D scans is reproducible. Findings of this study may be used to support future research measuring specific foot dimensions of children and adolescents with Down syndrome using 3D foot scans.

Keywords: Foot, Shoes, Down syndrome, Child, Adolescent, Foot deformities, 3-D image, Reproducibility of results

\section{Background}

Down syndrome is the most common chromosomal disorder [1], occurring in 1 in every 650 to 1000 live births [2]. Down syndrome affects multiple body systems including the nervous, cardiovascular and the musculoskeletal systems [3], resulting in intellectual and physical disability. Individuals with Down syndrome can have reduced physical fitness [4], ligamentous laxity, hypotonia, reduced lower limb muscle strength [5], less functional gait patterns [6] and gait instability [7].

Children and adolescents with Down syndrome commonly experience conditions associated with the foot that may impact their physical function. A population-based study involving 197 young individuals with Down syndrome reported $63 \%$ of individuals with Down syndrome were affected by a musculoskeletal condition of the foot [8]. At present, the exact cause of many of these conditions is not clearly understood. However, a contributing factor may be the unique foot shape of this population leading to difficulties in finding appropriately-fitting footwear. Children and adolescents with Down syndrome often have a flatter, shorter and broader foot [9], and are more likely to have foot deformities that includes lesser toe deformities and hallux valgus [9]. These deviations in foot shape are likely to contribute to the number of children and adolescents with Down syndrome who wear poorly-fitting footwear; 60 to $88 \%$ of children and adolescents with Down syndrome $[10,11]$ compared to $16 \%$ of typically developing children [11]. Poorly-fitting footwear can have adverse outcomes including the development of foot pain, which may lead to impaired health-related quality of life and altered gait patterns [12, 13]. Additionally, poorly-fitting footwear may contribute to reduced physical activity in children and adolescents with Down syndrome [13]. This is an issue for children and adolescents with Down syndrome because they have low levels of physical activity and are at greater risk of developing chronic health conditions as a result. It may also contribute to their reduced social participation. Further, having an intellectual disability can be an additional complicating factor as children and adolescents with Down syndrome may not always verbally communicate pain experienced to their caregivers despite being more sensitive to pain [14].

Given the high prevalence of poorly-fitting footwear and the potentially detrimental effects on health, improving footwear-fit for children and adolescents with
Down syndrome is important. Improving footwear fit may result in improved health-related quality of life and participation in physical activities. Additionally, improving the health of children and adolescents with Down syndrome may reduce burden to the health care system. Improving footwear fit can be achieved by designing footwear that can accommodate the unique foot shape of this population [12]. An essential initial step in designing footwear for children and adolescents with Down syndrome would be to capture the detailed foot dimensions of this population using reliable and valid methods. Although there is general consensus the foot shape of children and adolescents with Down syndrome differs to typically developing children, only one study [15] demonstrated the feet of young males with Down syndrome were shorter and narrower than age-matched peers, as measured using a podoscope. However, a limitation of this study is that only 2 foot dimensions (foot length and width) were measured using a 2-dimensional technique [8], which may not fully represent the complex shape of the foot. Three-dimensional (3D) scanning technology is a valid and reproducible means of obtaining detailed data on foot shape [16] and has been used to study the variations in foot shape in a number of different populations [17-19]. However, no studies have used 3D scanning to evaluate the foot dimensions of children and adolescents with Down syndrome, and the reproducibility of performing these measurements is unknown. Therefore, the aim of this study is to determine the reproducibility of measuring foot dimensions of children and adolescents with Down syndrome using 3D foot scanning.

\section{Methods}

This study is reported in accordance with Guidelines for Reporting Reliability and Agreement Studies [20].

\section{Study design}

Data were obtained from a previous feasibility study investigating the efficacy of custom-fitted footwear to increase physical activity levels in children and adolescents with Down syndrome [21]. The study was approved by the La Trobe University Human Ethics Committee (HEC16-027) and written informed consent was obtained from parents or guardians. Where appropriate, 
children and adolescents also provided written assent for participation prior to enrolment [21].

\section{Participants}

Participants were children and adolescents aged 5 to 17 years with Down syndrome. Participants were excluded if they had any health condition that may affect physical activity (e.g. inflammatory arthritis, subluxation etc.) as reported by their parents, or required the use of an ambulatory device (e.g. cane, crutches or walker). Participants were recruited through a member-based disability organisation for individuals with Down syndrome that was based in the community [21].

\section{Raters}

Two raters (NMH and AKB) performed the measurements for all foot scans, and measurements were repeated twice within 4 weeks to assess intra- and inter-rater reproducibility. Both raters were registered podiatrists with four and 14 years of clinical experience, respectively. Rater 1 had 3 months and rater 2 had 5 years of experience using 3D scans to measure foot dimensions, respectively.

\section{Measurements of participant characteristics}

Participants had their height and weight measured to calculate body mass index (BMI). Measurements were for the right foot only. Foot posture was assessed using two indices; the Foot Posture Index [22] and the Arch Index [23]. For both indices, higher positive scores indicate a flatter foot posture. The presence of lesser digital deformities (i.e. hammer, mallet and claw toes) was documented [10, 24]. The presence and severity of hallux valgus deformity was assessed using the Manchester scale [25]. The degree of deformity was graded on a scale of 0 to 3 (no deformity, mild, moderate and severe). Scores for hallux valgus were dichotomised, where scores of 0 and 1 were graded as absent and scores of 2 and 3 were graded as present [26].

\section{Scanning procedure}

Participants stood in a relaxed, full weight-bearing position and a 3D scan was taken of their right foot using the FotoScan 3D scanner (Precision 3D, Weston-supermare, UK). The FotoScan 3D device uses a fixed system of cameras and projectors to obtain images of the foot, that are automatically converted into a 3D model [27]. According to the manufacturer, the scans obtained with this system are accurate to within less than half a millimetre. The 3D foot scans were then exported as stereolithography (STL) files (Fig. 1). The 3D-Tool@ Version 13 (3D-Tool GmbH, Weinheim, Germany) was used to obtain all length, width and height measurements. For girth measurements, a cross-section of the foot at the

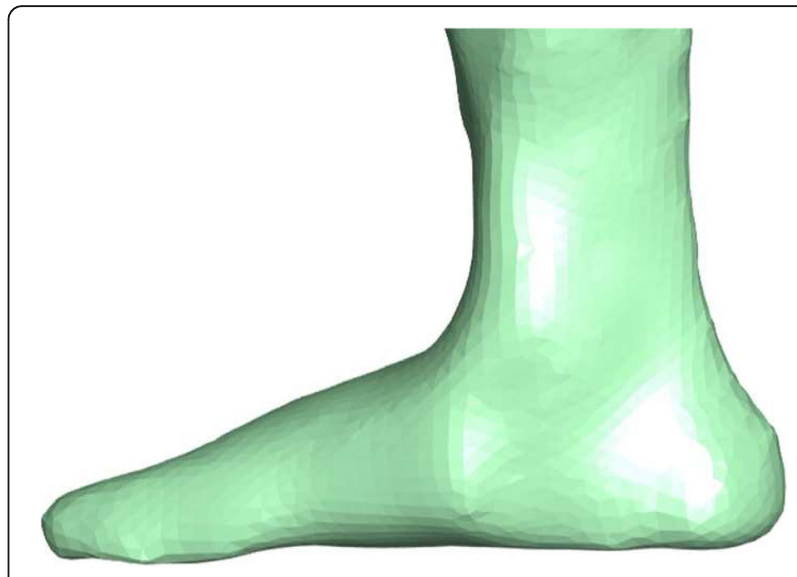

Fig. $13 \mathrm{D}$ model of right foot viewed from the medial aspect

relevant landmarks was created and exported as a drawing exchange format file. The perimeter of the crosssection was determined using Canvas $\odot 11$ software (ACD Systems International, Seattle, WA, USA).

\section{Measurement of foot dimensions}

Prior to data collection, pilot testing of the foot measurements was completed. A measurement technique protocol was developed (see Additional file 1). The protocol was piloted on the scans of five participants and the results were compared. Where there was substantial variability in results, the measurement technique was clarified between raters until consensus was reached. Each rater worked independently during data collection and compiled measurements on separate Excel spreadsheets (Microsoft ${ }^{\circ}$ Office 365, Microsoft, Redmond, WA, USA) on two separate occasions that were two weeks apart.

We measured 13 foot dimensions that are relevant to footwear manufacturing $[17,28,29]$. These were (Figs. 2 and 3):

1. Foot length: distance between foot end (pternion) and foot tip (anterior point of most protruding toe).

2. Ball of foot length: distance between foot end (pternion) and the first metatarsophalangeal protrusion.

3. Outside ball of foot length: distance between foot end (pternion) and the fifth metatarsophalangeal protrusion.

4. Diagonal foot width: connecting line between the first metatarsophalangeal joint and the fifth metatarsophalangeal joint.

5. Horizontal (orthogonal) foot width: orthogonal connection line starting at the first metatarsophalangeal joint to the outside curvature of the foot. 


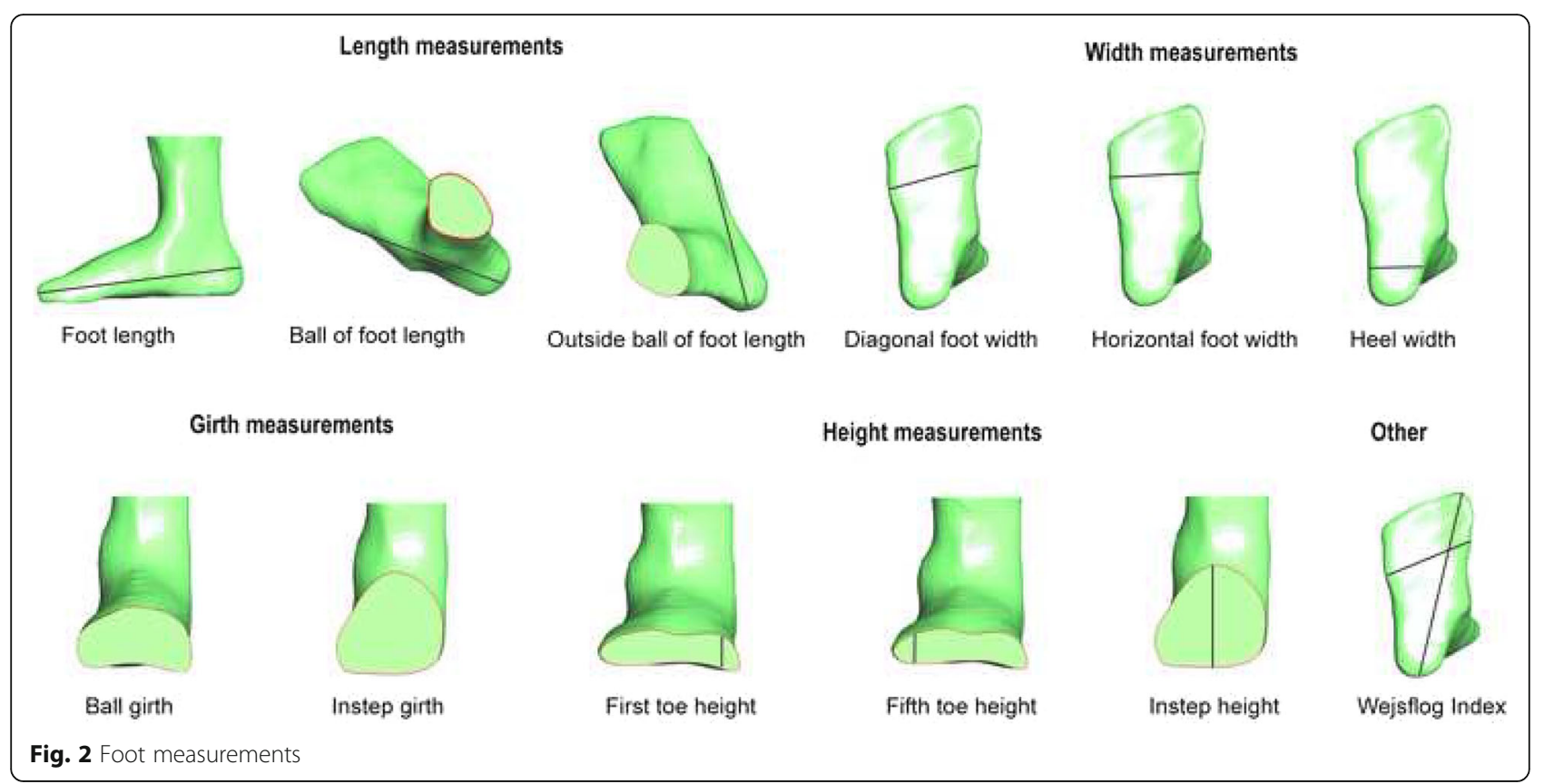

6. Heel width: maximum, orthogonal connection line starting at the medial side of the heel to the outside curvature of the heel.

7. Wejsflog Index: a ratio measurement of foot length to the diagonal forefoot width.

8. Ball girth: maximum circumference at the level of the first and the fifth metatarsophalangeal joint protrusion.

9. Instep girth: maximum circumference measured from the most plantar aspect of the foot to the most dorsal aspect of the foot, at the level of the navicular.

10. First toe height: maximum height of the hallux measured from the most plantar aspect of the hallux to the most dorsal aspect of the hallux.
11. Fifth toe height: maximum height of the fifth toe measured from the most plantar aspect of the fifth toe to the most dorsal aspect of the fifth toe.

12. Instep height: measured from the most plantar aspect of the foot to the most dorsal aspect of soft tissue (plantar foot end to the junction of shank and foot dorsum).

13. Forefoot shape: determined by categorising forefoot shape into three categories using the length of the digits. The categories were (i) first toe longest; (ii) second toe longest; and (iii) first and second toe length equal length.

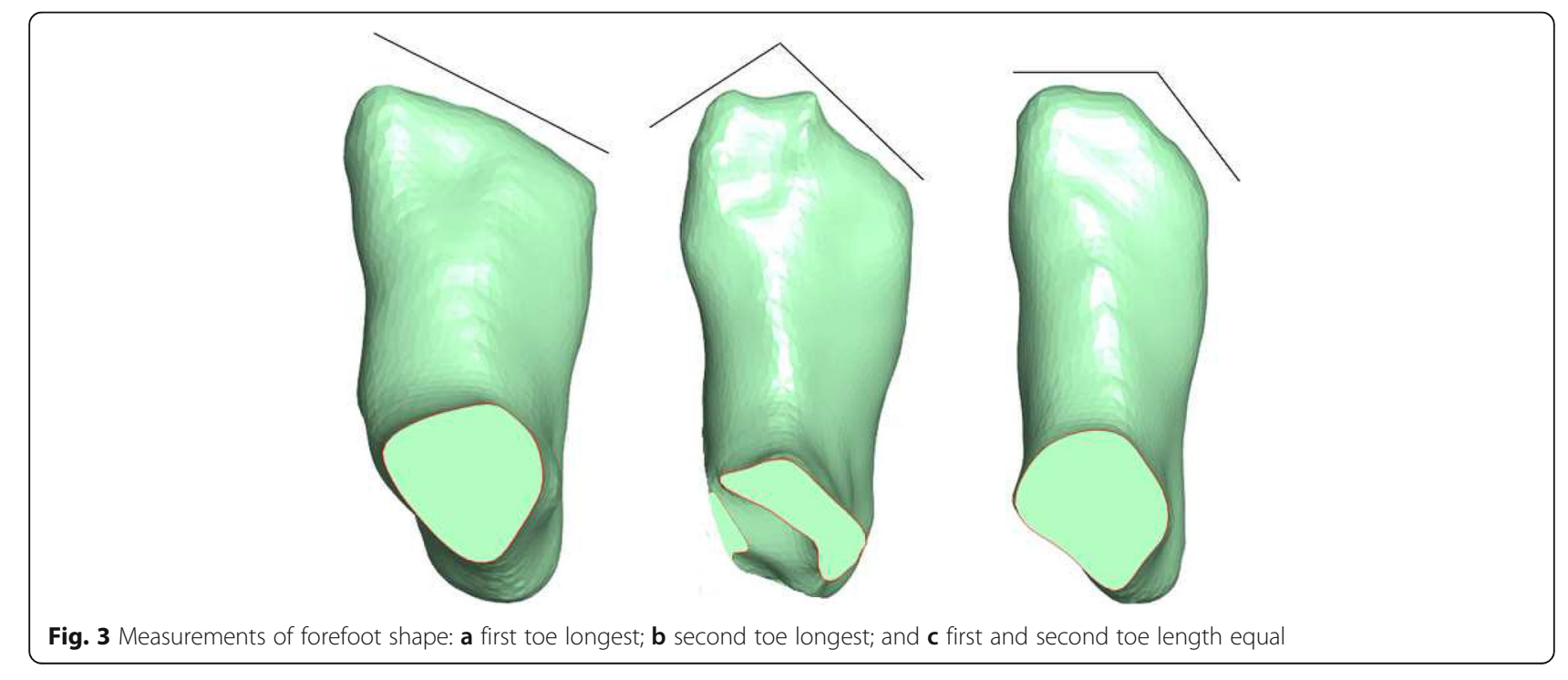




\section{Statistical analysis}

An a priori sample size estimation using the Power Analysis and Sample Size software (PASS 15 software, NCSS, LLC, Kaysville, UT, USA) for the ICC test, alpha of 0.05 , power 0.80 and ICC of 0.60 determined that a minimum sample size of 19 participants (foot scans) was necessary. However, as we had access to foot scans of 30 children and adolescents with Down syndrome, all scans were measured. Analyses were performed using IBM SPSS Statistics version 25.0 (IBM Corp, NY, USA) and STATA SE Version 14.2 (StataCorp, College Station, TX, USA), using the kappaetc module. To satisfy the independence assumption of statistical analysis, measurements from the right foot only were analysed [30]. For continuous data, data were assessed for normality using skewness and kurtosis tests, and data were found to be normally distributed. For continuous data, reliability was calculated using ICCs with 95\% confidence intervals (ICC [1, 2], consistency) [31]. Interpretation of ICCs were based on definitions provided by Portney and Watkins [32], where ICC values less than 0.5 was considered as poor, values between 0.5 to 0.75 was considered as moderate, values between 0.75 to 0.90 was considered as good and any value above 0.90 was considered as excellent reliability. For nominal data, reliability was determined by calculating Gwet's AC1 statistic [33] and results were interpreted according to Landis and Koch cut-offs, which are: less than zero was considered poor, 0 to 0.20 slight, 0.21 to 0.40 fair, 0.40 to 0.60 moderate, 0.60 to 0.80 substantial, 0.81 to 1.00 almost perfect reliability [34]. For continuous data, agreement was determined by calculating limits of agreement (LOA) [35]. For the calculation of LOAs, the presence of heteroscedasticity was assessed, and when present, ratio LOAs were calculated by taking the antilog of the calculated LOA values [36]. For nominal data, agreement was determined by using percentage agreement.

\section{Results}

Table 1 summarises the participant characteristics. There were 18 females and 12 males, with a mean (SD) age of 10.6 (3.9) years. The mean Foot Posture Index was +9 (1.8) and the mean Arch Index was 0.30 (0.05), indicating that on average, participants had a flat foot type. Hallux valgus was present in 4 participants (13\%), and 14 participants $(46 \%)$ had some degree of deformity of one of the lesser toes. No participants were excluded on the basis of requiring an ambulatory device.

\section{Intra-rater reproducibility}

Table 2 summarises the intra-rater reproducibility of all 13 foot measurements. The intra-rater reliability of rater 1 was good to excellent for 11 out of 13 measurements, with ICCs ranging from 0.76 to 0.99 . Fifth toe height and forefoot shape measurements had moderate reliability only $(\mathrm{ICC}=0.74$, Gwet's $\mathrm{AC} 1=$ 0.68 respectively). For rater 2, intra-rater reliability was good to excellent for all 13 measurements (ICCs/ Gwet's $\mathrm{AC} 1 \geq 0.83$ ).

There were 6 measurements (foot length, diagonal foot width, Wejsflog Index, first toe height, fifth toe height and instep height) for both raters and 1 measurement for Rater 2 (horizontal foot width) that exhibited narrow LOAs ranging from $-5.7 \mathrm{~mm}$ to 8.1 $\mathrm{mm}$. However, 5 measurements (ball of foot length, outside ball of foot length, heel width, ball girth, and instep girth) demonstrated wider LOAs ranging from $-13.5 \mathrm{~mm}$ to $16.3 \mathrm{~mm}$. For forefoot shape, agreement ranged from 73 to $87 \%$.

Table 1 Participant characteristics - values are mean (SD) unless otherwise stated

\begin{tabular}{lc}
\hline Characteristic & Mean (SD) \\
\hline Age, years & $10.6(3.9)$ \\
Females/males, $\mathrm{n}$ & $18 / 12$ \\
Height $(\mathrm{m})$ & $1.30(0.19)$ \\
Weight $(\mathrm{kg})$ & $40.1(21.4)$ \\
$\mathrm{BMI}^{1}\left(\mathrm{~kg} / \mathrm{m}^{2}\right)$ & $21.9(6.7)$ \\
Type of Down syndrome, $\mathrm{n}$ (Trisomy $21 /$ Translocation) & $27 / 3$ \\
Level of intellectual disability (unclear/mild/moderate/severe) & $9 / 12 / 9 / 0$ \\
Presence of hallux valgus, $\mathrm{n}(\%)$ & $4(13.0)$ \\
Presence of digital deformity, $\mathrm{n}(\%)$ & $14(46.0)$ \\
Foot Posture Index ${ }^{2}$ & $8.8(1.8)$ \\
Arch Index & \\
\hline
\end{tabular}

${ }^{1}$ Body mass index; ${ }^{2}$ Foot Posture Index; ${ }^{3}$ Arch Index. The Foot Posture Index scores range from -12 to +12 . Scores less than 1 indicate a supinated foot posture, scores between 1 and 7 indicate a normal foot posture, and scores greater than 7 indicate a pronated foot posture. Arch Index represents the ratio of the area of the middle third of a footprint to the entire footprint area, excluding the digits. Normal: 0.21 to 0.28 , high: $<0.21$ and low: $>0.28$ 
Table 2 Intra-rater reproducibility of foot measurements

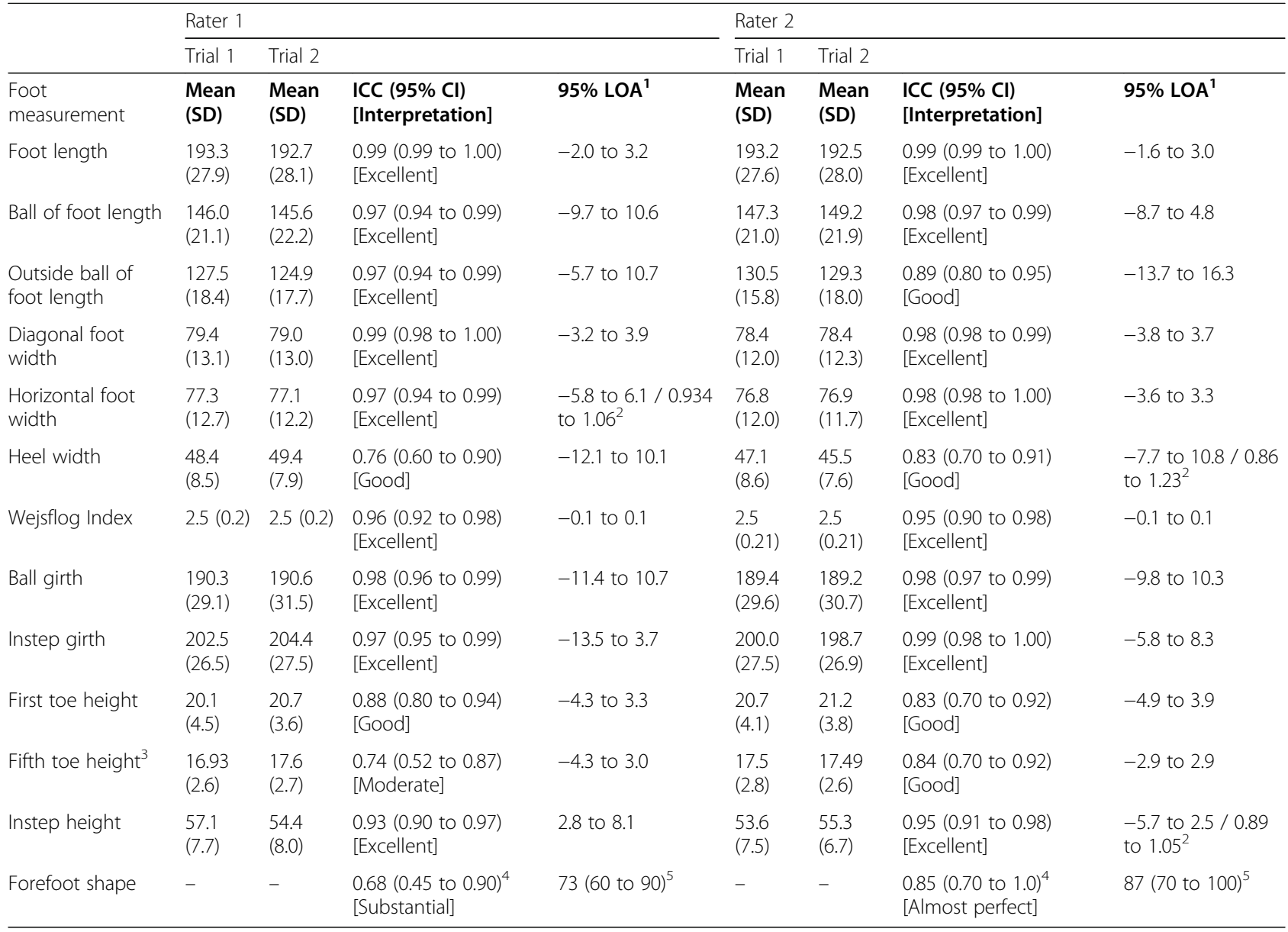

${ }^{1}$ Limits of agreement. ${ }^{2}$ Ratio limits of agreement also presented as measurement displays heteroscedasticity. ${ }^{3} 29$ scans were used due to the presence of artefacts. ${ }^{4}$ Gwet's AC1. ${ }^{5}$ Percentage agreement. Foot dimensions are measured in millimetres

\section{Inter-rater reproducibility}

Table 3 summarises the inter-rater reproducibility of all 13 foot measurements. The inter-rater reliability was good to excellent for all 11 measurements, with ICCs ranging from 0.89 to 0.99 . Fifth toe height and forefoot shape measurements had moderate reliability only ( $\mathrm{ICC}=0.73$ and Gwet's $\mathrm{AC} 1=0.77$, respectively).

Six measurements (foot length, diagonal foot width, horizontal foot width, Wejsflog Index, first toe height and fifth toe height) demonstrated narrow LOAs, ranging from $-4.4 \mathrm{~mm}$ to $5.8 \mathrm{~mm}$. However, the remaining 5 measurements (ball of foot length, outside ball of foot length, ball girth, instep girth and instep height) displayed relatively wider LOAs ranging from $-18.8 \mathrm{~mm}$ to $12.7 \mathrm{~mm}$. Percentage agreement for forefoot shape was $80 \%$.

\section{Discussion}

We found that the foot dimensions of children and adolescents with Down syndrome can be measured reliably from 3D foot scans. Our results indicate moderate to excellent reliability for all foot dimension measurements as demonstrated by high inter-rater ICC values or Gwet's $\mathrm{AC} 1$ values. However, the measurement of fifth toe height displayed poorer reliability. We observed some measurements (foot length, diagonal foot width, horizontal foot width, Wejsflog Index, first toe height and fifth toe height) had narrow LOAs indicating good agreement between raters, whereas others (ball of foot length, outside ball of foot length, ball girth, instep girth and instep height) displayed wider LOAs, suggesting relatively poorer agreement.

We observed differences in the performance of the two raters. The reliability for rater 1 was poorer than rater 2 for the measurements of heel width, fifth toe height and forefoot shape. The reason for this is unclear but could be that these measurements are more challenging to measure due to difficulty in locating the boundaries of these regions, particularly the toe region [16]. It is also possible that varying experience of the raters influenced the findings, since the reproducibility of rater 2 , who had 5 years of experience, was greater than rater 1 , who had 3 months' experience. This speculation is supported by previous 
Table $\mathbf{3}$ Inter-rater reproducibility of foot measurements

\begin{tabular}{|c|c|c|c|c|}
\hline Foot measurement & Rater 1 Mean (SD) & Rater 2 Mean (SD) & ICC (95\% CI) [Interpretation] & $95 \% \mathrm{LOA}^{1}$ \\
\hline Foot length & $193.3(27.9)$ & $193.2(27.6)$ & 0.99 (0.99 to 1.00) [Excellent] & -2.8 to $2.9 / 0.99$ to $1.01^{2}$ \\
\hline Ball of foot length & $146.0(21.1)$ & $147.3(21.0)$ & 0.97 (0.94 to 1.00) [Excellent] & -10.8 to 8.3 \\
\hline Outside ball of foot length & $127.5(18.4)$ & $130.5(15.8)$ & 0.89 (0.80 to 0.95 ) [Good] & -18.8 to 12.7 \\
\hline Diagonal foot width & $79.4(13.1)$ & $78.4(12.0)$ & 0.98 (0.96 to 0.99) [Excellent] & -3.8 to 5.8 \\
\hline Horizontal foot width & $77.3(12.7)$ & $76.8(12.0)$ & 0.98 (0.96 to 0.99) [Excellent] & -4.3 to 5.4 \\
\hline Heel width & $48.4(8.5)$ & $47.1(8.6)$ & 0.91 (0.81 to 0.95) [Excellent] & -5.9 to $8.6 / 0.89$ to $1.19^{2}$ \\
\hline Wejsflog Index & $2.4(0.2)$ & $2.5(0.2)$ & 0.90 (0.82 to 0.96 ) [Good] & -0.2 to 0.2 \\
\hline Ball girth & $190.3(29.1)$ & $189.4(29.6)$ & 0.99 (0.97 to 0.99) [Excellent] & -8.5 to 10.2 \\
\hline Instep girth & $202.5(26.5)$ & $200.0(27.5)$ & 0.98 (0.97 to 0.99) [Excellent] & -7.3 to 12.2 \\
\hline First toe height & $20.1(4.5)$ & $20.7(4.1)$ & 0.92 (0.84 to 0.96) [Excellent] & -3.9 to 2.9 \\
\hline Fifth toe height ${ }^{3}$ & $16.9(2.6)$ & $17.5(2.8)$ & 0.73 (0.50 to 0.86) [Moderate] & -4.4 to 3.3 \\
\hline Instep height & $57.1(7.7)$ & $53.6(7.4)$ & 0.90 (0.81 to 0.95 ) [Good] & -3.0 to 9.9 \\
\hline Forefoot shape & - & - & $0.77(0.58 \text { to } 0.96)^{4}$ [Substantial] & $80(65 \text { to } 95)^{5}$ \\
\hline
\end{tabular}

${ }^{1}$ Limits of agreement. ${ }^{2}$ Ratio limits of agreement also presented as measurement displays heteroscedasticity. ${ }^{3} 29$ scans were used due to the presence of artefacts. ${ }^{4}$ Gwet's AC1. ${ }^{5}$ Percentage agreement. Foot dimensions are measured in millimetres

work that has shown that rater experience and training is an important consideration in the reliability of measuring foot dimensions, particularly when it involves manual allocation of landmarks for calculating dimensions [16].

As this is the first study to investigate the reproducibility of the measurement of foot dimensions of children and adolescents using 3D scans, it is not possible to directly compare our findings. However, our findings are in general agreement with studies that investigated the reliability of measuring foot dimensions using adult foot scans where ICCs ranged from 0.82 to 0.99 [16, 27, 37-40].

Our findings relating to agreement allows for the interpretation of the acceptability of the reproducibility of the measurements. Where the LOA value is less than the minimally important difference for a measurement, the agreement could be considered acceptable. However, the value for the minimally important difference may vary depending on the context of the measurement. For example, for footwear fitting, it is common practice that foot length and foot width are measured in order to select an appropriately sized shoe. The International Organization for Standardization (2015) for footwear reports whole sizes (US and UK) differ in length by $8 \mathrm{~mm}$ [41], which indicates the reproducibility of the measurement of foot length is likely to be acceptable, as the range for the interrater LOA value for this measurement was $3.9 \mathrm{~mm}$. That is, it is less than the value that would necessitate a difference in shoe size $(8 \mathrm{~mm})$. In contrast, the acceptability of the measurement of horizontal foot width is questionable, as the range of the inter-rater LOA value was $9.7 \mathrm{~mm}$, which exceeds the standard $4.8 \mathrm{~mm}$ that defines a difference in shoe width [42]. The acceptability of the reproducibility of the remaining measurements (if used to guide the manufacture of footwear) for children and adolescents with Down syndrome is unclear, as shoe last dimensions for different shoe sizes is commercially sensitive information and not readily available.

Footwear-fit can be difficult in children and adolescents with Down syndrome because of variations to the shape of their feet. This study has shown measuring the foot dimensions from 3D foot scans of children and adolescents with Down syndrome can be done reproducibly. As they are reproducible, these measurements can be used with confidence in several settings. In a clinical setting, clinicians could perform measurements of foot dimensions to monitor and inform parents of their child's foot shape, and to provide guidance on appropriate footwear selection. In an applied research setting, the measurements may be used to determine detailed differences in foot dimensions of children and adolescents with Down syndrome, which may then be considered when manufacturing footwear for this population.

Our findings need to be interpreted in the context of the strengths and limitations of this study. We used two raters who worked independently throughout the data collection process in order to reduce the risk of bias, and we assessed both intra- and inter-rater reproducibility for completeness of results. In addition, both raters were podiatrists, who were experienced in foot and ankle measurements. However, the generalisability of the findings to raters from other professional backgrounds and/ or with different experience in measuring 3D foot scans needs further investigation. Finally, the measurements in this study were manually calculated according to definitions that were outlined in our methods, so findings may differ if dimensions are calculated by scanning software with pre-populated definitions of measurements that are different to the ones that we used. 


\section{Conclusions}

The measurement of specific foot dimensions of children and adolescents with Down syndrome using 3D scans is reproducible. The measurement described has multiple applications. In a clinical setting, clinicians can perform measurements of foot dimensions to monitor and inform parents of their child's foot shape, and to provide guidance on appropriate footwear selection. In an applied research setting, the measurements may be used to determine detailed differences in foot dimensions of children and adolescents with Down syndrome, which may then be considered when manufacturing footwear for this population.

\section{Supplementary information}

Supplementary information accompanies this paper at https://doi.org/10. 1186/s13047-020-00403-1.

Additional file 1. 3D foot scan measurement protocol

\section{Abbreviations}

2D: 2-dimensional; 3D: 3-dimensional; BMI: Body mass index; ICC: Intra-class coefficient; LOA: Limits of agreement

\section{Acknowledgements}

We acknowledge all participants and their parents for being involved in the original feasibility study in which this data was derived from. $\mathrm{NMH}$ is a recipient of an Australian Government Research Training Scholarship 2015. This work was supported by the La Trobe University Living with Disability Research Centre, as well as the La Trobe University Sport, Exercise and Rehabilitation Research Focus Area.

\section{Authors' contributions}

$\mathrm{NMH}, \mathrm{SEM}, \mathrm{KBL}$ and NS conceived the idea and design of the study. Collection and assembly of data was completed by NMH and AKB. Data analysis and interpretation was completed by NMH, SEM, KBL, HBM and NS. The manuscript was drafted by NMH, SEM, KBL, HBM and NS. All authors contributed substantially to the review and editing of the final manuscript. All author(s) read and approved the final manuscript.

\section{Funding}

$\mathrm{NMH}$ is a recipient of an Australian Government Research Training Scholarship 2015. This work was supported by the La Trobe University Living with Disability Research Centre.

\section{Availability of data and materials}

The datasets used and/or analysed during the current study are available from the corresponding author upon reasonable request.

\section{Ethics approval and consent to participate}

Ethical approval was obtained from the La Trobe University Human Ethics Committee (ethics approval number HEC16-027).

\section{Consent for publication}

Consent for publication was obtained from parents of participants. All authors have read and approved the final manuscript.

\section{Competing interests}

The authors declare that they have no competing interests.

\section{Author details}

'Discipline of Podiatry, School of Allied Health, Human Services and Sport, La Trobe University, Victoria 3086, Australia. ${ }^{2}$ Living with Disability Research Centre, School of Allied Health, Human Services and Sport, La Trobe University, Victoria 3086, Australia. ${ }^{3}$ La Trobe Sport and Exercise Medicine
Research Centre, School of Allied Health, Human Services and Sport, Victoria 3086, Australia. ${ }^{4}$ Discipline of Physiotherapy, School of Allied Health, Human Services and Sport, La Trobe University, Victoria 3086, Australia.

Received: 2 March 2020 Accepted: 26 May 2020

Published online: 04 June 2020

\section{References}

1. Oliver TR, Feingold $E$, Yu $K$, Cheung $V$, Tinker $S$, Yadav-Shah $M$, et al. New insights into human nondisjunction of chromosome 21 in oocytes. PLoS Genet. 2008:4(3):e1000033.

2. Bittles $A H$, Glasson EJ. Clinical, social, and ethical implications of changing life expectancy in Down syndrome. Dev Med Child Neurol. 2004;46(4):282-6

3. Bianca S. Non congenital heart disease aspects of Down's syndrome. Images Paediatr Cardiol. 2002:4(4):3-11.

4. Mendonca GV, Pereira FD, Fernhall B. Reduced exercise capacity in persons with Down syndrome: cause, effect, and management. Ther Clin Risk Manag. 2010;6:601-10.

5. Foley C, MacDermott E, Killeen O. Musculoskeletal anomalies in a national cohort of children and adolescents with trisomy 21. Pediatr Rheumatol Online J. 2014;12:P160.

6. Galli M, Rigoldi C, Brunner R, Virji-Babul N, Giorgio A. Joint stiffness and gait pattern evaluation in children with Down syndrome. Gait Posture. 2008; 28(3):502-6.

7. Cimolin V, Galli M, Grugni G, Vismara L, Albertini G, Rigoldi C, et al. Gait patterns in Prader-Willi and Down syndrome patients. J Neuroeng Rehabil. 2010;7:28.

8. Pikora TJ, Bourke J, Bathgate K, Foley K-R, Lennox N, Leonard H. Health conditions and their impact among adolescents and Young adults with Down syndrome. PLoS One. 2014;9(5):e96868.

9. Concolino D, Pasquzzi A, Capalbo G, Sinopoli S, Strisciuglio P. Early detection of podiatric anomalies in children with Down syndrome. Acta Paediatr. 2006;95(1):17-20.

10. Lim PQ, Shields N, Nikolopoulos N, Barrett J, Evans A, Taylor N, et al. The association of foot structure and footwear fit with disability in children and adolescents with Down syndrome. J Foot Ankle Res. 2015;8:4.

11. Calvo-Lobo C, Ramos Garcia A, Losa Iglesias ME, Lopez-Lopez D, RodriguezSanz D, Romero-Morales C, et al. The relationship between shoe fitting and foot health of persons with Down syndrome: a case control study. Int J Environ Res Public Health. 2018;15(5):983.

12. Buldt AK, Menz HB. Incorrectly fitted footwear, foot pain and foot disorders: a systematic search and narrative review of the literature. J Foot Ankle Res. 2018;11:43.

13. Shields N, Lim P, Wollersheim D, Nikolopoulos N, Barrett J, Evans A, et al. Do foot posture, deformity, and footwear fit influence physical activity levels in children with Down syndrome? A prospective cohort study. J Intellect Disabil Res. 2017;42(4):332-8.

14. McGuire BE, Defrin R. Pain perception in people with Down syndrome: a synthesis of clinical and experimental research. Front Behav Neurosci. 2015; 9:194.

15. Puszczałowska-Lizis E, Nowak K, Omorczyk J, Ambroży T, Bujas P, Nosiadek L. Foot structure in boys with Down syndrome. Biomed Res Int. 2017;7047468.

16. Lee $Y-C$, Lin G, Wang M-JJ. Comparing 3D foot scanning with conventional measurement methods. J Foot Ankle Res. 2014;7:44.

17. Krauss I, Grau S, Mauch M, Maiwald C, Horstmann T. Sex-related differences in foot shape. Ergonomics. 2008;51(11):1693-709.

18. Lee YC, Kouchi M, Mochimaru M, Wang MJ. Comparing 3D foot shape models between Taiwanese and Japanese females. J Hum Ergol (Tokyo) 2015:44(1):11-20

19. Wunderlich RE, Cavanagh PR. Gender differences in adult foot shape: implications for shoe design. Med Sci Sports Exerc. 2001;33(4):605-11.

20. Kottner J, Audigé L, Brorson S, Donner A, Gajewski BJ, Hróbjartsson A, et al. Guidelines for reporting reliability and agreement studies (GRRAS) were proposed. J Clin Epidemiol. 2011;64(1):96-106.

21. Hassan NM, Shields N, Landorf KB, Buldt AK, Taylor NF, Evans AM, et al. Efficacy of custom-fitted footwear to increase physical activity in children and adolescents with Down syndrome (ShoeFIT): randomised pilot study. Disabil Rehabil. 2019:1-10 [Epub ahead of print]. 
22. Redmond AC, Crosbie J, Ouvrier RA. Development and validation of a novel rating system for scoring standing foot posture: The Foot Posture Index. Clin Biomech (Bristol, Avon). 2006;21(1):89-98.

23. Cavanagh PR, Rodgers MM. The arch index: a useful measure from footprints. J Biomech. 1987;20(5):547-51.

24. Menz HB, Lord SR. Foot pain impairs balance and functional ability in community-dwelling older people. J Am Podiatr Med Assoc. 2001;91(5):222-9.

25. Garrow AP, Papageorgiou A, Silman AJ, Thomas E, Jayson MI, Macfarlane GJ. The grading of hallux valgus. The Manchester scale. J Am Podiatr Med Assoc. 2001;91(2):74-8.

26. Menz HB, Munteanu SE. Radiographic validation of the Manchester scale for the classification of hallux valgus deformity. Rheumatology (Oxford). 2005; 44(8):1061-6.

27. Menz HB, Auhl M, Ristevski S, Frescos N, Munteanu SE. Evaluation of the accuracy of shoe fitting in older people using three-dimensional foot scanning. J Foot Ankle Res. 2014;7:3.

28. Goonetilleke R. The science of footwear. 1st ed ed. Boca Raton: CRC Press; 2013

29. Lee $Y C$, Wang MJ. Taiwanese adult foot shape classification using 3D scanning data. Ergonomics. 2015;58(3):513-23.

30. Menz HB. Two feet, or one person? Problems associated with statistical analysis of paired data in foot and ankle medicine. Foot (Edinb). 2004;14(1):2-5.

31. Koo TK, Li MY. A guideline of selecting and reporting Intraclass correlation coefficients for reliability research. J Chiropr Med. 2016;15(2):155-63.

32. Portney L, Watkins M. Foundations of clinical research. Applications to practice. 2 ed. Upper Saddle River, NJ. : Prentice Hall Health; 2000

33. Wongpakaran N, Wongpakaran T, Wedding D, Gwet KL. A comparison of Cohen's kappa and Gwet's AC1 when calculating inter-rater reliability coefficients: a study conducted with personality disorder samples. BMC Med Res Methodol. 2013;13:61.

34. Landis JR, Koch GG. The measurement of observer agreement for categorical data. Biometrics. 1977;33(1):159-74.

35. Bland JM, Altman DG. Measuring agreement in method comparison studies. Stat Methods Med Res. 1999;8(2):135-60.

36. Nevill A, Atkinson G. Assessing agreement between measurements recorded on a ratio scale in sports medicine and sports science. Br J Sports Med. 1997:31:314-8

37. De Mits S, Coorevits P, De Clercq D, Elewaut D, Woodburn J, Roosen P. Validity and reliability of the Infoot 3D foot digitizer for rheumatoid arthritis patients. J Am Podiatr Med Assoc. 2009;101(3):198-207.

38. Robinette KM, Daanen HA. Precision of the CAESAR scan-extracted measurements. Appl Ergon. 2006;37(3):259-65

39. Seminati E, Canepa Talamas D, Young M, Twiste M, Dhokia V, Bilzon JLJ. Validity and reliability of a novel 3D scanner for assessment of the shape and volume of amputees' residual limb models. PLoS One. 2017;12(9): e0184498.

40. De Mits S, Coorevits P, De Clercq D, Elewaut D, Woodburn J, Roosen P. Reliability and validity of the Infoot 3D foot digitizer for normal healthy adults. Footwear Sci. 2010;2(2):65-75.

41. International Organization for Standardization. ISO/TS 19407. Footwear Sizing - Conversion of sizing systems. 2015.

42. Brannock Device. 2019. [Available from: http://www.brannock.com].

\section{Publisher's Note}

Springer Nature remains neutral with regard to jurisdictional claims in published maps and institutional affiliations.

Ready to submit your research? Choose BMC and benefit from:

- fast, convenient online submission

- thorough peer review by experienced researchers in your field

- rapid publication on acceptance

- support for research data, including large and complex data types

- gold Open Access which fosters wider collaboration and increased citations

- maximum visibility for your research: over $100 \mathrm{M}$ website views per year

At BMC, research is always in progress.

Learn more biomedcentral.com/submissions 